\title{
Case Profiles of Noninvasive Functional Brain Imaging as an Alternative to Cortical Stimulation Mapping
}

\author{
Roozbeh Rezaie ${ }^{1,4}$, James W Wheless ${ }^{1,4}$ Frederick A Boop ${ }^{2,4 *}$ and Shalini Narayana ${ }^{1,3,4}$ \\ ${ }^{1}$ Department of Pediatrics, Division of Pediatric Neurology, University of Tennessee Health Science Center, USA
}

${ }^{2}$ Department of Neurosurgery, University of Tennessee Health Science Center, USA

${ }^{3}$ Department of Anatomy and Neurobiology, University of Tennessee Health Science Center, USA

${ }^{4}$ Neuroscience Institute, Le Bonheur Children's Hospital, USA

Submission:December 14, 2017; Published: February 21, 2018

*Corresponding author: Frederick A Boop, Department of Neurosurgery, University of Tennessee Health Science Center, 49 N. Dunlap St, 3rd Floor, Memphis, TN 38105, USA, Fax: 901-287-5325, Tel: 901-448-6375; Email: frederickboop@gmail.com

\begin{abstract}
Cortical stimulation mapping (CSM) has long been considered the gold-standard for localizing the eloquent cortex in the presurgical evaluation of patients with epilepsy and brain tumors. In recent years, however, the introduction of non-invasive brain mapping techniquesnamely functional magnetic resonance imaging (fMRI), magnetoencephalography (MEG) and transcranial magnetic stimulation (TMS)- have presented an alternative to CSM for identifying the central sulcus and specifying the motor cortex, as well as approximating expressive and receptive language cortices, particularly in cases where invasive monitoring may not be practical. Moreover, the efficacy of the non-invasive methods as a viable substitute for CSM has been attested to in a number of comparison studies in clinical cohorts. In this paper, we present two case studies which demonstrate the utility of the non-invasive functional mapping techniques (MEG and TMS) during presurgical evaluation. Specifically, we first report an instance of preserved sensorimotor function within an area of frontal polymicrogyria coincident with the ictal onset zone in a female with intractable symptomatic partial epilepsy; second, we present findings from a female patient where language localized to the boundary of a left temporal lobe ganglioglioma. In both cases, the outcome of the non-invasive techniques was deemed to be of sufficient utility to defer surgery and seek alternative treatment measures, in order to preserve function and reduce morbidity.
\end{abstract}

Keywords: Presurgical functional mapping; Non-invasive; Epilepsy; Brain tumor; CSM; MEG; TMS; fMRI

Abbreviations: CSM: Cortical stimulation mapping; fMRI: Functional magnetic resonance imaging; MEG: Magnetoencephalography; TMS: Transcranial Magnetic Stimulation

\section{Introduction}

Presurgical localization of eloquent cortex in epilepsy and brain tumor patients has traditionally been performed using cortical stimulation mapping (CSM), either intra- or extraoperatively, using subdural electrodes. While CSM is considered the "gold standard" in presurgical functional mapping, recent literature has highlighted the merits of non-invasive brain mapping techniques as possible alternatives to CSM, for outlining the cortical mapping of the cortical regions underlying motor, somatosensory and language functions [1]. These noninvasive functional imaging techniques are: functional magnetic resonance imaging (fMRI), most readily available across most surgery centers, magneto encephalography (MEG) and, more increasingly, transcranial magnetic stimulation (TMS). Evidence of the clinical efficacy of these procedures [2-5], used alone or in combination, has presented the possibility of substituting them for CSM, particularly in view of some practical limitations associated with the latter, including cost, morbidity, seizure provocation and limited patient compliance due to age or developmental delay. In this report, we highlight the utility of non-invasive brain mapping methods, in two presurgical cases, where clinical recommendations to pursue alternative treatments to surgical intervention were facilitated by combined concordance of MEG and TMS information.

\section{Patient Histories}

\section{Patient 1}

The patient, admitted for Phase 1 epilepsy surgery evaluation, was a 19 year-old right-handed female with 


\section{Open Access Journal of Neurology \& Neurosurgery}

intractable symptomatic partial epilepsy of right frontal lobe origin, encephalopathy characterized by borderline impairment of intellectual function, and left upper extremity monoparesis secondary to right hemisphere closed-lip schizencephaly. Routine MRI of the brain revealed a right frontal schizencephalic cleft and polymicrogyria along and adjacent to the margins of the cleft, as well as absence of the septum pellucidum, and possible optic nerve hypoplasia, most likely to be in the spectrum of septo-optic dysplasia. Interictally, prolonged video electroencephalography (EEG) monitoring revealed sharp waves over both frontal polar and frontal head regions. While partial onset seizures showed no distinct EEG changes, those going on to secondary generalization were characterized by bilateral rhythmic theta frequencies that progressed to bilateral spikes and then bilateral polyspikes and slow waves. Interictal and ictal single-photon emission computed tomography (SPECT) were obtained, subtracted and co-registered to the MRI where they showed an area of increased uptake in the right frontal lobe just adjacent to the anterior aspect of the cleft and slight involvement of the anterior superior temporal lobe on the right.

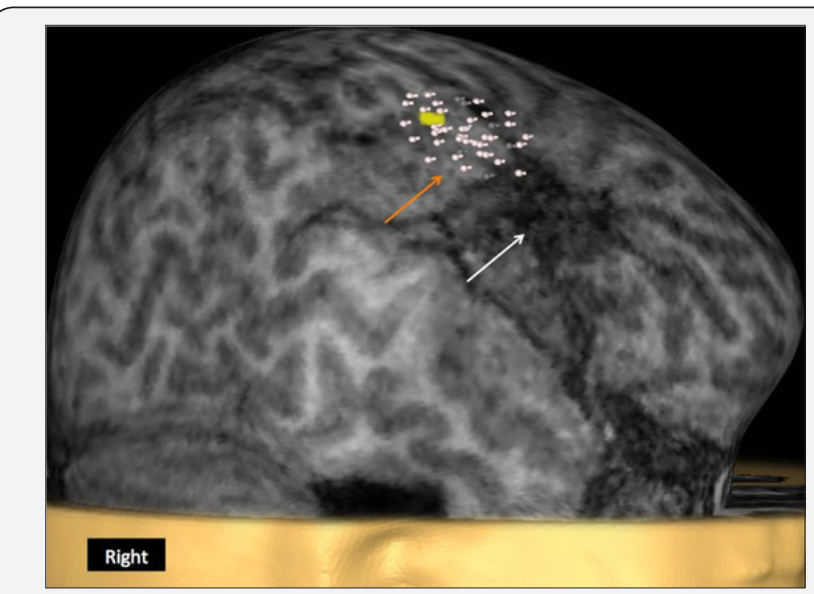

Figure 1 : Anatomical and Functional mapping with TMS and MEG in patient 1. MRI demonstrates the right frontal schizencephalic cleft (white arrow) and polymicrogyria (orange arrow) along and adjacent to the margins of the cleft. The cortical locations where TMS elicited motor evoked potentials in the contralateral hand muscles are shown as white pegs projected onto the patient's MRI. TMS localized the motor cortex to the area of polymicrogyria. The source of activity associated with the peak of the early latency response to the pneumatic stimulation of left index finger was localized by MEG is shown in yellow. The somatosensory cortex was localized to the post central gyrus along the posterior margin of polymicrogyria.

In consideration of the ictal onset zone, as well as lesional focus, identified by diagnostic imaging, non-invasive functional mapping was requested to approximate the extent of the sensory and motor cortices, prior to recommendation of surgical intervention. Initially, the extent of the upper and lower extremities was assessed using navigated single pulse TMS, applied to bilateral primary motor cortices while recording electromyogram from abductor pollicis brevis, abductor digiti minimi, and tibialis anterior muscles (6). Motor evoked potentials (MEP) were elicited following TMS of both primary hand motor cortices and resting motor thresholds (rMT) were determined using the relative frequency method. The location of left primary hand motor cortex was determined to be normal. The primary hand motor cortex in the right hemisphere was displaced antero-inferiorly and was located over the area of polymicrogyria (Figure 1). Stimulation of medial frontal gyrus resulted in MEPs in tibialis anterior muscle and the primary leg motor cortex was found to be normally located in both hemispheres. Subsequently, identification of the primary somatosensory cortices was achieved with MEG, using a series of pneumatically driven mechanical taps applied to the fingertip of the patient's left index finger via a balloon diaphragm [7]. The primary somatosensory cortex in the right hemisphere was localized to the post central gyrus by modelling the source of activity associated with the peak of the early latency response contralateral to the side of stimulation (Figure 1).

While convergence of evidence indicated that seizures were originating from the area of right closed lip schizencephaly and polymicrogyria that was lining the cleft, the preserved sensorimotor function in the polymicrogyria was demonstrated with MEG and TMS suggested that resection in order to improve seizure control would likely result in the loss of all motor and sensory function of the left side. As such, the patient sought alternative treatment options for seizure control, namely vagus nerve stimulation in combination with pharmacotherapy.

\section{Patient 2}

The patient was an 8 year-old right handed-female admitted for pre-operative evaluation for a ganglioglioma of the left temporal lobe. Prior pathological diagnosis was suspicious but not diagnostic of an non-invasive presurgical functional mapping 5 infiltrating glioma, and a previous subtotal resection brought the patient's seizures under medical control, though follow-up MRI showed progression of the residual tumor. Upon admission, routine MRI revealed an approximately $14 \mathrm{~mm}$ nodular enhancing lesion in the posterior aspect of the left superior temporal gyrus, with surrounding areas of leptomeningeal enhancement, with imaging characteristics suggestive of a neoplastic entity such as ganglioglioma. Other than the lepto meningeal enhancement in the adjacent sylvian fissure, there is no suspicious enhancement distant from the primary lesion. To assess the risk of prospective language deficits in the event of surgical resection, the patient was recommended to undergo comprehensive expressive and receptive language testing using TMS and MEG. Hemispheric dominance for expressive language was assessed in the context of disruption of speech during $5 \mathrm{~Hz}$ rapid TMS [6] applied as patient performed a picture-naming task. TMS applied to the middle part of superior and middle temporal gyrii, temporoparietal junction, and the inferior frontal gyrus in the left hemisphere resulted in disruption of speech in the form of speech arrest, slowing and slurring (Figure 2, right panel). The stimulation of right-sided speech and language regions also resulted in speech impairment including speech arrest, sematic errors, and slowing and slurring. Localization of language 


\section{Open Access Journal of Neurology \& Neurosurgery}

specific cortex and subsequent determination of hemispheric dominance for receptive language with MEG was performed in the context of a continuous auditory word recognition protocol previously described [3] in a large-scale studies detailing specific procedures for determining hemispheric dominance for language functions. Single-trial MEG segments in response to $110-125$ stimulus presentations were averaged and receptive language cortex was identified by evoked activity sources computed during the late portion ( $>200 \mathrm{~ms}$ ) of the event-related field waveform, falling within fronto-temporal, temporo-parietal and mesial temporal regions, for each hemisphere $[3,8]$.

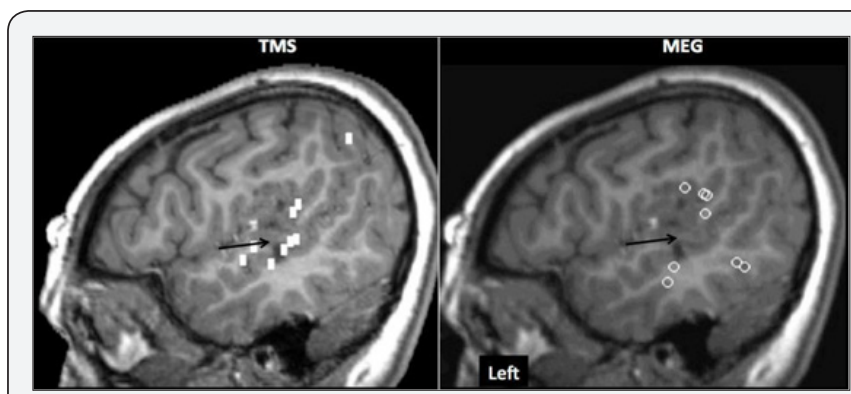

Figure 2 : Anatomical and Functional mapping with TMS and MEG in patient 2. MRI demonstrates ganglioglioma including previous resection in the left temporal lobe (black arrows). Left panel: The cortical locations where TMS elicited speech disruption in the left hemisphere are shown as white squares projected onto the patient's MRI. TMS localized language areas along the margins of the tumor. Right panel: The receptive language areas in the left temporal lobe identified by MEG during continuous auditory word recognition protocol are shown as white circles. The evoked activity sources were also localized along the margins of the tumor.

Both TMS and MEG converged to demonstrate critical language regions in close proximity to the ganglioglioma, approximating Wernicke's area, deemed indispensable for semantic processing (Figure 2). To mitigate the potential for post-operative language deficit, and in view of prior surgical intervention, it was felt that the risk of tumor resection at present exceeded the benefits, and chemotherapy and radiation therapy were recommended as further treatment measures.

\section{Discussion}

Beyond their theoretical contributions in systems neuroscience, the fundamental utility of non-invasive brain mapping techniques such as fMRI, MEG and TMS, is emphasized by their ability to outline the neural circuitry

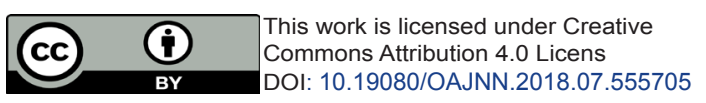

underlying sensorimotor and language processes. From a practical perspective, reliable and expedient localization of the aforementioned functions are often critical to the process of pre surgical planning in patients with epilepsy and brain tumors, especially in cases where resective surgery carries the prospect of functional morbidity. Indeed, comparisons of the efficacy of non-invasive methods against conventional invasive monitoring suggest that the former may be a reasonable substitute for the latter, as it relates to reducing morbidity, facilitating surgical planning, and enhancing surgical outcome. Despite their promise, however, it should be emphasized that further methodological refinements of the non-invasive methods are necessary to improve their predictive power, especially for language mapping, if they are to fully replace their invasive counterparts in clinical practice.

\section{Referances}

1. Papanicolaou AC, Rezaie R, Narayana S, Choudhri AF, Wheless JW, et al. (2014) Is it time to replace the wada test and put awake craniotomy to sleep? Epilepsia 55(5): 629-632.

2. Janecek JK, Swanson SJ, Sabsevitz DS, Hammeke TA, Raghavan M, et al. (2013) Language lateralization by fMRI and Wada testing in 229 patients with epilepsy: rates and predictors of discordance. Epilepsia 54(2): 314-322.

3. Papanicolaou AC, Simos PG, Castillo EM, Breier JI, Sarkari S, et al. (2004) Magnetocephalography: a noninvasive alternative to the Wada procedure. J Neurosurg 100: 867-876.

4. Picht T, Krieg SM, Sollmann N, Rosler J, Niraula B, et al. (2013) A comparison of language mapping by preoperative navigated transcranial magnetic stimulation and direct cortical stimulation during awake surgery. Neurosurgery 72(5): 808-819.

5. Tarapore PE, Tate MC, Findlay AM, Honma SM, Mizuiri D, et al. (2012) Preoperative multimodal motor mapping: a comparison of magnetoencephalography imaging; navigated transcranial magnetic stimulation; and direct cortical stimulation. J Neurosurg 117(2): 354362 .

6. Narayana S, Papanicolaou AC, McGregor A, Boop FA, Wheless JW (2015) Clinical applications of transcranial magnetic stimulation in pediatric neurology. Journal of Child Neurology 30(9): 1111-24.

7. Castillo EM, Simos PG; Wheless JW, Baumgartner JE, Breier JI, et al. (2004) Integrating sensory and motor mapping in a comprehensive MEG protocol: clinical validity and replicability. Neuroimage 21: 973983.

8. Simos PG, Sarkari S, Castillo EM, Billingsley-Marshall RL, Pataraia E, et al. (2005) Reproducibility of measures of neurophysiological activity in Wernicke's area: a magnetic source imaging study. Clin Neurophysiol 116(10): 2381-2391.

\begin{tabular}{|l|}
\hline \multicolumn{1}{|c|}{ Your next submission with Juniper Publishers } \\
will reach you the below assets \\
- Quality Editorial service \\
- Swift Peer Review \\
- Reprints availability \\
- E-prints Service \\
- Manuscript Podcast for convenient understanding \\
- Global attainment for your research \\
- Manuscript accessibility in different formats \\
( Pdf, E-pub, Full Text, Audio) \\
- Unceasing customer service \\
Track the below URL for one-step submission \\
https://juniperpublishers.com/online-submission.php \\
\hline
\end{tabular}

